\title{
Molecular Cloning and Expression of Bottlenose Dolphin (Tursiops truncatus) Interleukin-8
}

\author{
Takuya ITOU $^{1)}$, Yuuki YOSHIDA ${ }^{1)}$, Yoko SHOJI ${ }^{1)}$, Hitoshi SUGISAWA ${ }^{1)}$, Tomoko ENDO ${ }^{2)}$ and Takeo SAKAI ${ }^{1)}$ \\ ${ }^{1)}$ Department of Preventive Veterinary Medicine and Animal Health, Nihon University School of Veterinary Medicine, 1866 Kameino, \\ Fujisawa, Kanagawa 252-8510 and ${ }^{2}$ Shinagawa Aquarium, 3-2-1 Katsushima, Shinagawa-ku, Tokyo 140-0012, Japan
}

(Received 3 March 2003/Accepted 11 August 2003)

ABSTRACT. The bottlenose dolphin interleukin (IL)-8 cDNA was molecularly cloned. The dolphin IL-8 has an open reading frame of 303bp encoding 101 amino acids. The homology of the amino acid sequence with that of other species was: sheep, 89.1\%; cattle, 88.1\%; pig, $85.1 \%$; dog, $85.1 \%$; horse, $79.2 \%$; human, $74.5 \%$; and macaque, $72.3 \%$. The amino acid sequence suggested that dolphin IL-8 was a CXC chemokine. The recombinant dolphin IL-8 protein was recognized with anti-ovine IL-8 monoclonal antibody. KEY WORDS: bottlenose dolphin, cDNA cloning, IL-8.

Interleukin (IL)-8 is a CXC chemokine with neutrophil chemotactic activity and is produced by macrophages and various tissue cells at the inflammation site. Chemokines stimulate the extracellular migration of neutrophils, which play an important role in the early host defense response to infection. IL-8 has a variety of physiologic effects not only on neutrophils, but also on T lymphocytes, basophils, skin keratinocytes, and melanocytes. IL- 8 enhances the host defense response with respect to neutrophils by increased chemotaxis, production of reactive oxygen species, and adherence to vascular endothelial cells, and mobilization from the bone marrow to the periphery $[3,8,11]$. IL- 8 has been isolated and purified from the supernatants of human peripheral mononuclear cells stimulated with lipopolysaccharide $[22,23]$. Molecular cloning studies have also been performed in other mammals, such as cattle and sheep, and in chickens, flounder, and rainbow trout [7, 10, 12-17, 21]. Continuous intravenous infusion of IL- 8 causes destruction of lung alveoli, and intraarticular administration causes destruction of synovial membranes. Other studies have reported the involvement of IL-8 with rheumatoid arthritis and angiogenesis in tumor growth. Thus, IL- 8 is involved not only in enhancing early host defense responses, but also in the pathogenesis of many disorders $[9,11]$. A decrease in host defense response and poor environmental conditions can often lead to the development of opportunistic infections in cetaceans [1]. Studies to date have shown that gene sequences for IL- $1 \alpha$, IL- $1 \beta$, IL- 1 ra, IL-4, TNF- $\alpha$, and IFN- $\gamma$ in bottlenose dolphins are highly homologous to the corresponding genes in ruminants $[4-6,19]$. There is, however, some suggestion of differences in the production of reactive oxygen species between dolphin and bovine neutrophils [18]. As an attempt to clarify the immune mechanism in marine mammals, we isolated and characterized the IL-8 gene from bottlenose dolphins and produced a recombinant IL-8 protein.

Peripheral blood samples were obtained from the tail vein of clinically healthy bottlenose dolphins bred by the Shina- gawa Aquarium for display and educational use. Blood samples were collected in heparinized vacuum tubes. The mononuclear cells were isolated by density gradient centrifugation according to the method described by Inoue et al. [4]. Stimulated culture was conducted with RPMI 1640 medium containing $10 \%$ fetal calf serum. The cell suspension was adjusted to $1 \times 10^{7}$ cells $/ \mathrm{ml}$, and concanavalin A (Wako Pure Chemical Industries, Ltd., Osaka, Japan) was added to a final concentration of $2.5 \mu \mathrm{g} / \mathrm{ml}$. In 24-well flatbottom plates, a total volume of $1 \mathrm{~m} l$ was cultured at $37^{\circ} \mathrm{C}$ for $6 \mathrm{hr}$. After culture, the total RNA was extracted from the cells with ISOGEN (Nippon Gene, Toyama, Japan).

Synthesis and amplification of the cDNA encoding for dolphin IL-8 was performed by the method described by Inoue et al. [6]. The primers used to amplify the dolphin IL8 gene were 5' - CAAGAGCCAGGAAGAAACC-3' (IL8F) and 5'-CACTGATTCTTGGATACCA-3' (IL-8R). These primers were designed based on highly conserved regions in the IL-8 gene of humans (accession No. Y00787), sheep (accession No. S74436), and macaque (accession No. U19849). PCR was done for 35 cycles: denaturation, $94^{\circ} \mathrm{C}$ for $1 \mathrm{~min}$; annealing, $55^{\circ} \mathrm{C}$ for $1 \mathrm{~min}$; extension, $74^{\circ} \mathrm{C}$ for 1 min. Amplified cDNA was electrophoresed on 2\% agarose gel and then purified with a QIA Quick Gel Extraction Kit (QIAGEN, Gmbh, Germany). The purified cDNA was cloned with a pMOSBlue Blunt Ended Cloning Kit (Amersham Biosciences Corp., New Jersey, U.S.A.). For target colonies, insertion was confirmed by direct PCR with the above primers. The nucleotide sequence for the region coding for dolphin IL-8 protein was determined with T7 and U19 primers and a Thermo Sequenase Cy5.5 Dye Terminator Cycle Sequencing Kit (Amersham Biosciences Corp.) and Gene Rapid SEQ $4 \times 4$ Personal Sequencing System (Amersham Biosciences Corp.) in accordance with the accompanying manuals. Three clones had the same nucleotide sequence. Homology of the nucleotide sequence with the IL-8 genes of horse (AF062377), macaque (U19851), human (Y00787), cat (AF158598), dog (D14285), pig 
(X61151), sheep (S74436) and cattle (S82598) was confirmed with the dolphin sequence by using FASTA 3.0 (W. R. Pearson). Multialignment was performed with CLUSTAL X 1.8 (F. Jeanmougin). A phylogenetic tree generated by the neighbor joining method was drawn with Tree View 1.6 (Molecular Evolution). The amino acid sequence was determined from the nucleotide sequence with GENETYX-WIN Version 5.0 and compared with other species.

The mature dolphin IL- 8 cDNA was obtained by PCR with primers: 5'-GCTGGATCCGCAGTTCTATCAAGA3' (IL-8 protein-F: BamHI site is shown in bold) and 5'GAGCTCGAGTCATGGATCTTTCTT-3' (IL-8 protein-R: XhoI site is shown in bold), which were determined by sequencing. The PCR product was digested with Bam HI and $X h o I$ under the conditions recommended by the manufacturer (TaKaRa, Tokyo, Japan). The digested fragments were ligated into the $B a m \mathrm{HI} / X h o I$ restriction sites of the glutathione-S-transferase (GST) fusion plasmid vector, pGEX4T-1 (Amersham Biosciences Corp.) with a DNA Ligation Kit Ver. 2 (TaKaRa). E. coli (BL21) was transformed with the resulting plasmid DNA and grown in LB medium containing $50 \mu \mathrm{g} / \mathrm{m} l$ of ampicillin. Isopropyl- $\beta$-Dthiogalactopyranoside (IPTG) was added to a final concen- tration of $1 \mathrm{mM}$, followed by culture for an additional $2 \mathrm{hr}$. The culture was centrifuged and the pellet was resuspended in $10 \mathrm{~m} l$ of PBS and lysed by sonication. The crude lysate was centrifuged and the supernatant was collected as the $E$. coli sonicated products. To isolate the recombinant dolphin (rdo) IL- 8 protein, affinity purification was performed by the column method, as reported by Smith et al. [20], with GST Purification Modules (Amersham Biosciences Corp.). The purified rdoIL-8 protein was confirmed by SDS polyacrylamide gel electrophoresis (PAGE) and Western blotting with an anti-ovine IL-8 monoclonal antibody (Chemicon International Inc., California, U.S.A.).

The dolphin IL- 8 nucleotide sequence determined by cloning and sequencing and the putative amino acid sequence were deposited in the GenBank Data Base (accession No. AB096002). Analysis showed that the dolphin IL8 gene had 303 base pairs encoding 101 amino acids. It is a simple protein with a molecular weight of 9,168 Da and without potential $N$-linked glycosylation sites. Amino acids 34, 36, 61 and 77 in the presumptive sequence of bottlenose dolphin IL-8 are conserved as cysteine residues (Fig. 1). The homology of the dolphin IL-8 gene nucleotide sequence with other species was: cattle, $92.4 \%$; sheep, $91.1 \%$; pig, $87.6 \%$; cat, $86.8 \%$; dog, $85.8 \%$; human, $82.9 \%$; horse,

\begin{tabular}{|c|c|}
\hline dolphin & 1: MTSKLAIAIIAAFIL-SAA---ICKAAVISRMTSEIRCOCINIHS-----TPF-HPKFIR \\
\hline cattie & 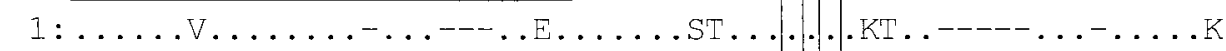 \\
\hline sheep & 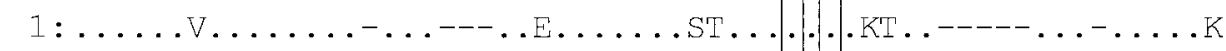 \\
\hline oig & 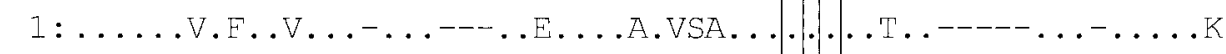 \\
\hline dog & $1: \ldots \ldots \vee \ldots \ldots V \cdot-\ldots--\ldots \mathrm{E} \ldots \ldots \mathrm{VS} \ldots . \cdot \cdot \cdot \mathrm{KT} \ldots----\ldots-\ldots \mathrm{Y} \cdot \mathrm{K}$ \\
\hline at & 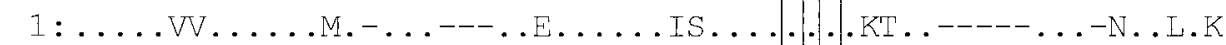 \\
\hline orse & 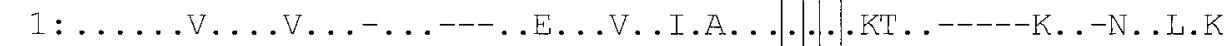 \\
\hline uman & 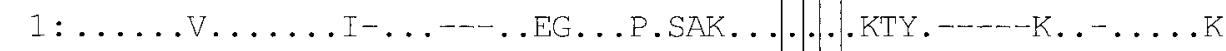 \\
\hline acaque & $1: \ldots \ldots V \ldots \ldots \ldots-\ldots-\ldots$ EG . . P.SAK ... $. . K T Y . \cdots-K \ldots-\ldots K$ \\
\hline icken & 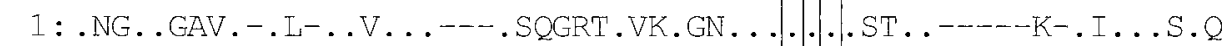 \\
\hline amprey & 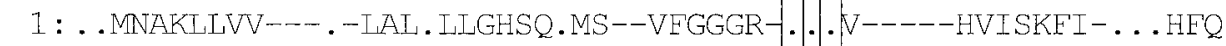 \\
\hline flounder & 1:--MSSRVIVV.VMV.LAS--IAISE.VS.RSLGVS.H.R.].ETE.RP-IGRY - - . - . K \\
\hline olphin & 51 : ELRVIESGPHCENSEI IVKLVNGKEV-CTINPKEKWVQKV---V-QIFLKRAEKK--D-P- \\
\hline attle & 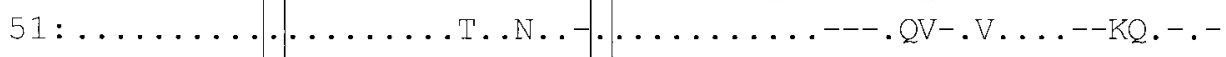 \\
\hline 1eep & 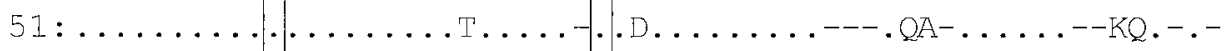 \\
\hline $\lg$ & 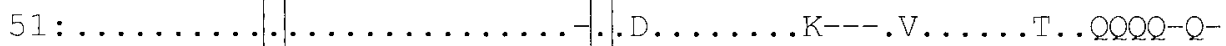 \\
\hline og & 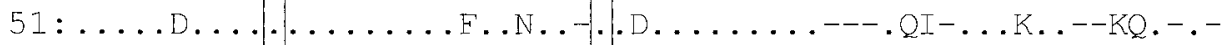 \\
\hline at & 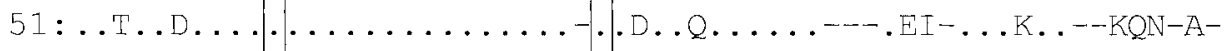 \\
\hline orse & 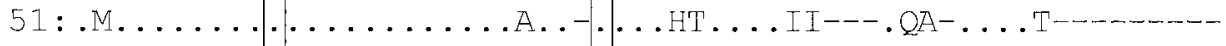 \\
\hline numan & 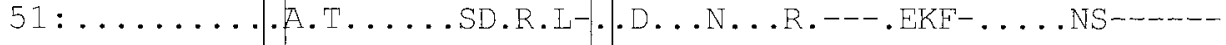 \\
\hline lacaque & 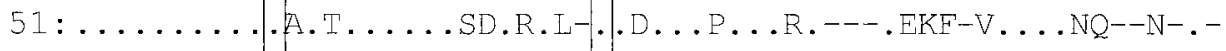 \\
\hline nicken & 50:DVKLTP.....K.V...AT.KD.R.....D.TAP...LI---.KATMA.A-QINSDA-.I \\
\hline mprey & 48: TME. . PQSSN.|K.V. . . TMKSTNNQI... . DAP . R. . ISHIIDGAQTP----KSTQ-- \\
\hline Lunder & 50: SVEI.SPNS. .DKT . .AAT.KDTGVEL...D.EAP. . KR. INKLISKRRLSRWREMGSEAV \\
\hline
\end{tabular}

Fig. 1. Alignment of deduced amino acid sequences of IL-8 from dolphin and several other species. The amino acid residues identical to the dolphin sequence are indicated by dots. Where the amino acids are absent, this is denoted by a dash. The conserved Cys residuesare delineated by solid boxes. The deduced signal sequence is underlined. 

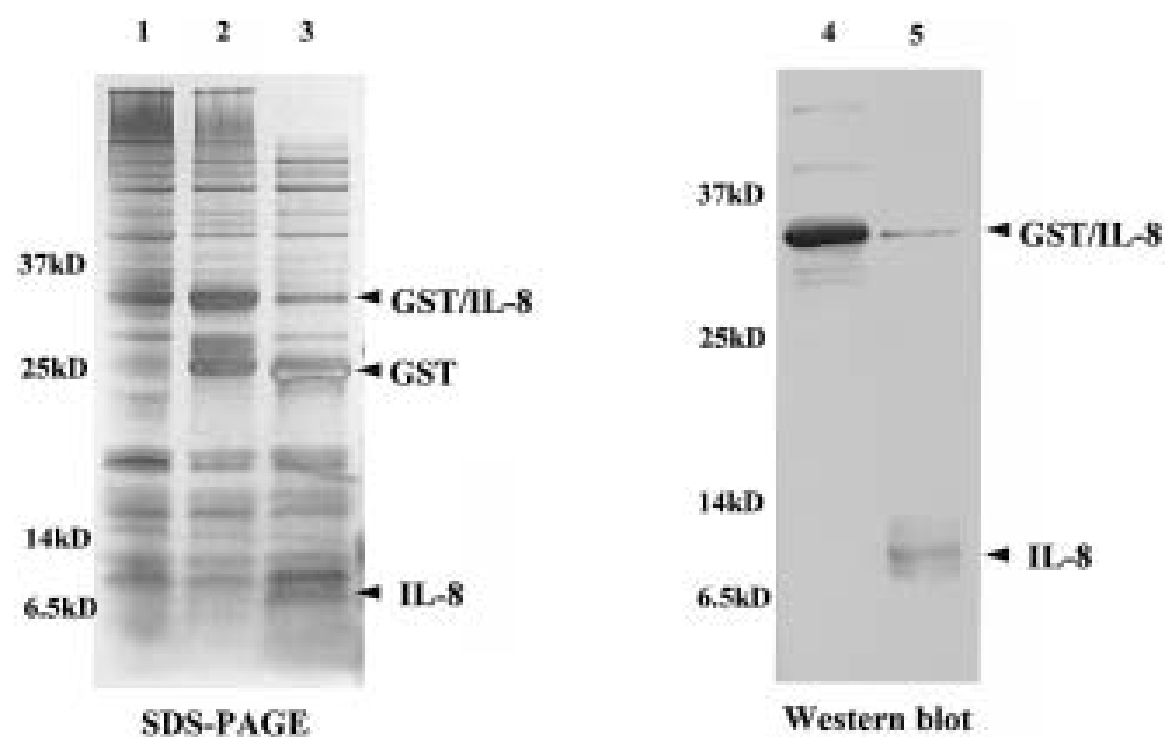

Fig. 2. SDS-PAGE and Western blot analysis of recombinant dolphin IL-8 expressed in E. coli. Lane 1: soluble fraction from culture with IPTG induction. Lane 2: GST/rdoIL-8 fusion protein. Lane 3: rdoIL-8 protein after thrombin cleavage. Western blot analysis with monoclonal antiovine IL-8 antibody (Lanes 4 and 5). Lane 4: GST/rdoIL-8 fusion protein. Lane 5: rdoIL-8 after thrombin cleavage.

$82.8 \%$; and macaque, $81.8 \%$. The homology of the amino acid sequence with other species was: cattle, $88.1 \%$; sheep, $89.1 \%$; pig, $85.1 \%$; dog, $85.1 \%$; cat, $80.2 \%$; human, $74.5 \%$; horse, $79.2 \%$; and macaque, $72.3 \%$. As reported for other dolphin cytokines [4-6, 19], our results showed a high degree of homology with ruminants. Analysis by means of the phylogenetic tree of dolphin IL-8 showed that it belonged to a different cluster than that of human and macaque. There was a closer relation to ruminants (data not shown).

SDS-PAGE showed bands for the GST/rdoIL-8 fusion protein at $34 \mathrm{kDa}, \mathrm{GST}$ after cleavage by thrombin at 25 $\mathrm{kDa}$, and the rdoIL-8 at $9 \mathrm{kDa}$ (Fig. 2). Western blotting with anti-ovine IL-8 antibody showed a band for the rdoIL8 with specific reactivity at $9 \mathrm{kDa}$.

IL-8 has a specific secretory protein signal sequence. The precursor form of IL- 8 is known to be converted to the mature form of IL- 8 by cleavage of this signal sequence. The region encoding dolphin IL-8 has a 22-amino-acid (66 bp) signal sequence at the 5' end. This suggests that the mature form of dolphin IL- 8 has 80 amino acids (240 bp). The CXC chemokines have four cysteine residues that are highly conserved and are characterized by a single amino acid between the number 1 and 2 cysteines (CXC motif) [11]. In the amino acid sequence of bottlenose dolphin IL8 , amino acids $34,36,61$ and 77 are conserved as cysteine residues, a typical structure of a CXC cytokine. Human IL8 has a beta-sheet structure with two disulfide bonds between the four cysteine residues. The C-terminal end starting at $\mathrm{Asn}^{83}$ assumes an alpha-helical structure. At high solution concentrations, there is also dimerization by virtue of hydrogen bonding through part of the beta-sheet structure
$[2,11]$. The mature form of bottlenose dolphin IL-8 also has four cysteine residues and thus probably has two disulfide bonds. Analysis of the stereostructure with GENETYXWIN suggests that the C-terminal end starting at Gln ${ }^{86}$ assumes an alpha-helical structure. SDS-PAGE of the rdoIL-8 protein produced by the $E$. coli expression system confirmed a band at about $9 \mathrm{kDa}$. This is similar to the molecular weight of mature IL- 8 reported in humans. In addition, Western blotting of the rdoIL-8 protein showed specific reactivity with anti-ovine IL- 8 monoclonal antibody. This antibody has reactivity with ovine, bovine, and caprine IL-8. These findings support the results for the homology of the nucleotide and amino acid sequences.

In conclusion, the presumptive amino acid sequence and stereostructure of dolphin IL-8 was similar to human IL-8. Moreover, the antigenic property of rdoIL- 8 was very close to that of artiodactyl IL-8. Our findings indicate that the protein structure of mammalian IL-8 is similar.

ACKNOWLEDGEMENTS. This research was financially supported by a Grant-in-Aid from the Ministry of Education, Science, Sports, and Culture of Japan and by a Sasakawa Scientific Research Grant from The Japan Science Society.

\section{REFERENCES}

1. Dunn, J. L. 1990. pp. 73-87. In: Handbook of Marine Mammal Medicine (Dunn J. L. ed.) CRC Press, Florida.

2. Eric, T. B., Weber, I. T., Charles, R., Xuan, J. C., Appella, E., Yamada, M., Matsushima, K., Edwards, B. F., Clore, G. M. and Gronenborn, A. M. 1991. Proc. Natl. Acad. Sci. U.S.A. 88: 502-509. 
3. Harada, A., Sekido, N., Kuno, K., Akiyama, M., Kasahara, T., Nakanishi, I., Mukaida, N. and Matsushima, K. 1993. Int. Immunol. 5: 681-690.

4. Inoue, Y., Itou, T., Sakai, T. and Oike, T. 1999. J. Vet. Med. Sci. 61: 693-696.

5. Inoue, Y., Itou, T., Oike, T. and Sakai, T. 1999. J. Vet. Med. Sci. 61: 939-942.

6. Inoue, Y., Itou, T., Jimbo, T., Syouji, Y., Ueda, K. and Sakai, T. 2001. Vet. Immunol. Immunopathol. 78: 131-141.

7. Ishikawa, J., Suzuki, S., Hotta, K., Hirota, Y., Mizuno, S. and Suzuki, K. 1993. Gene. 131: 305-306.

8. Iwabe, T., Harada, T., Tsudo, T., Nagano, Y., Yoshida, S., Tanikawa, M. and Terakawa, N. 2000. J. Clin. Endocrinol. Metab. 85: 824-829.

9. Koch, A. E., Polverini, P. J., Kunkel, S. L., Harlow, L. A., DiPietro, L. A., Elner, V. M., Elner, S. G. and Strieter, R. M. 1992. Science 258: 1798-1801.

10. Lee, E. Y., Park, H. H., Kim, Y. T. and Choi, T. J. 2001. Gene 274: $237-243$.

11. Mager, T. 1998. The Molecular Biology of Cytokines, Jhon Wiley \& Sons Ltd, Chichester.

12. Massion, P. P., Hebert, C. A., Leong, S., Chan, B., Inoue, H., Grattan, K., Sheppard, D. and Nadel, J. A. 1995. Am. J. Physiol. 268: 85-94.
13. Matsushima, K., Morishita, K., Yoshimura, T., Lavu, S., Kobayashi, Y., Lew, W., Appella, E., Kung, H. F., Leonard, E. J. and Oppenheim, J. J. 1988. Exp. Med. 167: 1883-1893.

14. Morsey, M. A., Popowych, Y., Kowalski, J., Gerlach, G., Godson, D., Campos, M. and Babiuk, L. A. 1996. Microbial. Pathogenesis 20: 203-212.

15. Mukaida, N., Harada, A., Yasumoto, K. and Matsushima, K. 1992. Mircobiol. Immunol. 36: 773-789.

16. Mukaida, N., Shiroo, M. and Matsushima, K. 1989. J. Immunol. 143: 1366-1371.

17. Seow, H. F., Yoshimura, T., Wood, P. R. and Colditz, I. G.. 1994. Immunol. Cell Biol. 72: 398-405.

18. Shiraishi, R., Itou, T., Sugisawa, H., Shoji, Y., Endo, T. and Sakai, T. 2002. J. Vet. Med. Sci. 64: 711-714.

19. Shoji, Y., Inoue, Y., Sugisawa, H., Itou, T., Endo, T. and Sakai, T. 2001. Vet. Immunol. Immunopathol. 82: 183-192.

20. Smith, D. B. and Johnson, K. S. 1988. Gene 67: 31-40.

21. Villinger, F., Brar, S. S., Mayne, A., Chikkala, N. and Ansari, A. A. 1995. J. Immunol. 155: 3946-3954.

22. Yoshimura, T., Matsushima, K., Oppenheim, J. J. and Leonard, E. J. 1987. J. Immunol. 139: 788-793.

23. Yoshimura, T., Matsushima, K., Tanaka, S., Robinson, E. A., Appella, E., Oppenheim, J. J. and Leonard, E. J. 1987. Proc. Natl. Acad. Sci. U.S.A. 84: 9233-9237. 